\title{
EMI-FREE DC-DC BUCK CONVERTER FOR AEROSPACE
} APPLICATIONS

\author{
Jithu Unnikrishnan ${ }^{1}$, O M Saji Chandrachood ${ }^{2}$, A.K Prakash ${ }^{3}$, K K Mukundan ${ }^{4}$ \\ ${ }^{1}$ PG Student, [Wireless Technology], ${ }^{3}$ Professor, Dept. of ECE, Toc H Institute of Science and Technology, Cochin, \\ Kerala, India \\ ${ }^{2,4}$ Scientist/Engineer, 'SD', ADRFD/RFSG/AVN, Vikram Sarabhai Space centre, Thiruvananthapuram, Kerala, India
}

\begin{abstract}
Modern electronic systems require tiny, high-quality, less weight, reliable and efficient power supplies. Linear power regulator whose principle of operation is based on a voltage or current divider is inefficient sometimes. This is because their output voltage is smaller than the input voltage and also they require low frequency $(50 \mathrm{or} 60 \mathrm{~Hz})$ transformers and filters. Their main area of application is at lower power levels; but at higher levels, switching regulators are used. They use semiconductor switches for the purpose of turning off and on states. Because there is a power loss in those states, switching regulators can achieve high energy conversion efficiencies and that is the major advantage. Power electronic systems such as switching power supplies are accounted as noise source for their sensitive circuits. EMI caused by the converters can disturb the normal operation of the supply and other systems by coupling with the nearby devices. Hence the overall performance of the system is reduced Major focus is done on the radiated EMI mitigation for the power converters in which the main standard is the compliance with the EMC standard to ensure the proper operation of the converter and nearby systems. Buck converter is a step-down converter which is one of the most widely used topologies among the different DC-DC topologies. In the forward topology, we are providing the galvanic isolation which isolates the functional sections of the electrical systems to prevent the current flow. No direct conduction is permitted. In this paper, different topologies are discussed and implemented which can reduce the Radiated Emission (RE) and MILSTD 461/462 will be the underlying EMC standard.
\end{abstract}

Keywords: Radiated Emission (RE), soft switching, snubber, forward converter

\section{INTRODUCTION}

Electro Magnetic Interference (EMI) is a disturbance that affects an electrical circuit by the coupling of the electromagnetic field from an external source; the coupling of the field can be either through conduction or radiation. This disturbance may interrupt or degrade the normal operation of the electrical circuits and hence it is considered as an unwanted characteristic. These effects can range from a simple degradation of data to the total loss of information. And Electro Magnetic Compatibility (EMC) is the capability of an electrical circuit to operate in an electromagnetic interference environment. Since the earliest days of radio communication, the negative effects of the intentional and unintentional transmission interference have been felt and the need to manage the radio frequency became clear. Later in 1983,a meeting of the IEC(International Electrotechnical Commission) made rise to the EMC standard CISPR(Comite International Special Dees Perturbations Radio electrique to deal with the emerging problem of EMI. From there on more and more standards came like FCC, MIL which were possessing particular rules and regulations to maintain EMC

DC-DC power converters which convert sources of direct current from one voltage level to the other. In aerospace applications, they are widely used to provide power to the flight and satellite packages. There are different converter topologies are used and buck converter is one of them which is used for stepping down the voltage. In the buck topology, the grounds are joined together. So it gets shorted. Hence due to noise, it gets affected from other systems. As a purpose of study we have taken forward topology which is the isolated form of buck. It uses a transformer to decrease the output voltage. In power supplies. Two prominent types of EMI are conducted and radiated EMI. In this paper we are focused only on the radiated emission, i.e. above $30 \mathrm{MHz}$ noise. Different topologies are discussed which can capable of mitigating the Radiated Emission (RE).

\section{RELATED WORKS}

In order to reduce the electromagnetic interference of the converter, a number of solutions have been proposed in the literature survey. The authors have addressed an improved soft switching forward converter. The proposed converter is constructed by using non dissipative snubbers in parallel with the main switch and output diode of the conventional forward topology. Due to the use of the energy recovery snubbers in the primary and secondary, the novel converter achieves zerovoltage and zero-current switching (ZVS and ZCS) for all 
switching devices without any losses like switching losses and output diode recovery losses[3]. Generally, RC or RCD snubbers are used in the primary and secondary sides of the forward converter in order to absorb the energy stored in the transformer leakage inductance, and to suppress the surge voltage. But, these converter topologies can't be used for high operating frequencies because this absorbed energy is dissipated in the snubber resistor, when the switch is in turnon or turn-off. To overcome this drawback and achieve high efficiency in high power and high frequency applications, the use of non-dissipative snubbers is very critical. In this paper, an improved soft switching forward converter utilizing an energy recovery snubber is mentioned.

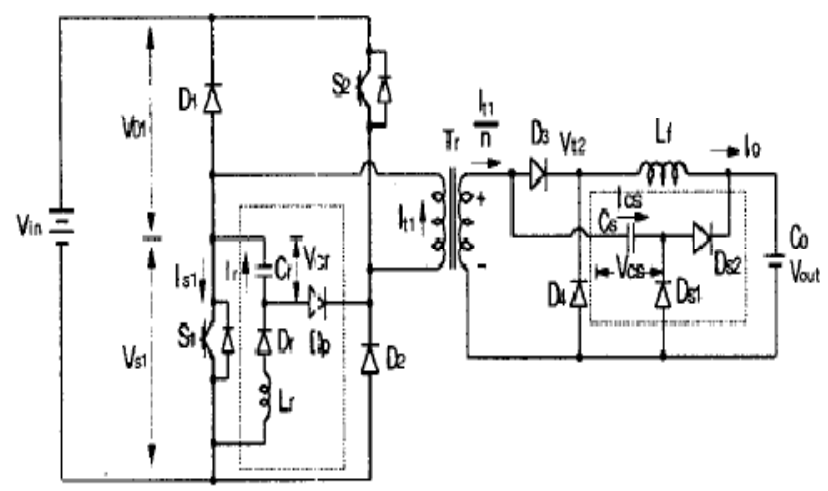

Fig-1: Forward converter with energy recovery snubber[3]

In [9], the authors found a new technique, known as spreadspectrum clock generation (SSCG), for reducing the magnitude of radiated emission from devices with digital clock signals. To calculate the radiated emissions from such devices, they have modelled the radiating geometry and computed the radiated field at a multitude of frequencies by using an electromagnetic field solver based on the method of moments (MoM).Modulation of the clock frequency creates side-bands, spreading the harmonic peaks which leads to the EMI suppression of the emission spectrum. And it is observed that upto $30 \mathrm{~dB}$ reduction in the radiated emission levels.

Another method for reducing the radiated emissions of an electronic device by frequency modulating (FM) the system clock This proposed method is referred to as Spread Spectrum Clock Generation, or SSCG[6]. A unique waveform is used to frequency modulate a digital clock signal results in a frequency plot with sideband harmonics that are spreaded nearly uniform in amplitude when measured with an EMI receiver. It affects like spreading the energy of a discrete frequency harmonic over a wider bandwidth, and thereby reducing the amplitudes of theharmonics. With spread spectrum ideology, the energy at each of these harmonics is spread over a wider bandwidth, thereby reducing the peak amplitude. In addition, the bandwidth over which each harmonic is spread increases linearly with frequency so that the attenuation provided by Spread spectrum clocking also increases linearly with frequency.

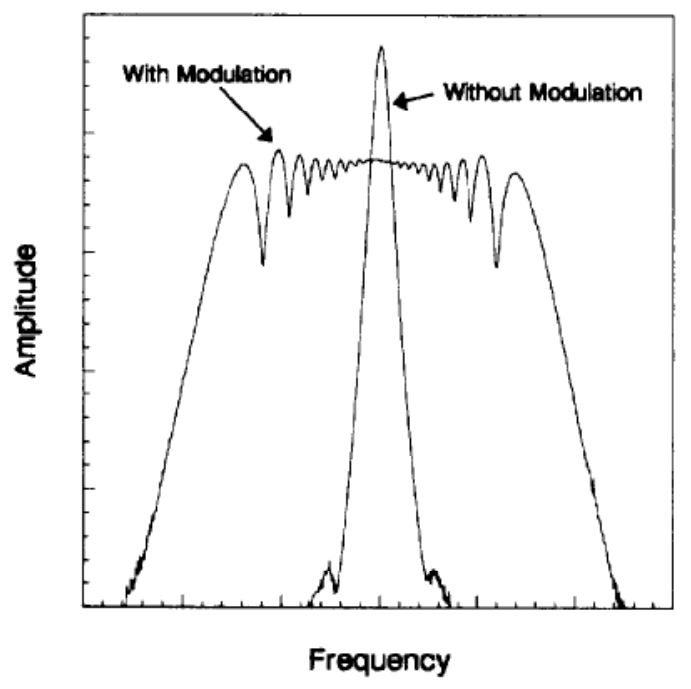

Fig-2: Frequency domain representation at a harmonic of a trapezoidal clock signal with and without SSCG.[6]

In [4], the authors have depicted a novel method, dithered timing spread spectrum clock(DT-SSC) for the suppression of the electromagnetic radiation from the high-speed digital system. The proposed dithered timing spread spectrum clock has more effective suppression than the conventional spread spectrum clock (SSC). The conventional spread spectrum clock is realized by controlling the period or the frequency using a phase locked loop(PLL). Because of the inherent jitter of the phase locked loop, the attenuation of the spectrum is minimized and the realization is very difficult at the higher clock frequencies near the $\mathrm{GHz}$ range. In this paper, they have proposed a novel method. a dithered timing spread spectrum clock. The dithered timing spread spectrum clock is executed by controlling the transition timing-difference and a delayer is responsible for that. The proposed dithered timing spread spectrum clock has more effective suppression of the electromagnetic disturbance from the high-speed digital system than the conventional spread spectrum clock.

\section{PROPOSED SOLUTION}

Due to the operation of the power converter, the electromagnetic interference is generated. Since we are concentrated in the radiated emission, we will be discussing some of the solutions to mitigate that. Radiated EMI comprises of two things, electric and magnetic fields. The electric field strength is directly proportional to the voltage of the circuit, frequency of operation, and antenna's effective length. On the other side, The strength of the magnetic field is directly proportional to the current flowing through the circuit, frequency of operation and the antenna loop's effective area. Since circuit parameters and operation frequency are fixed in 
terms of a converter's operating characteristics, the only variable term is the length of the power line, or the enclosed loop area of the power line's return path. Therefore, radiated EMI can be minimized by physically locating the noisegenerating source as close to its source and load as possible. However, such a compact assembly is rarely possible for mechanical reasons.

\subsection{Soft Switching}

Large $\mathrm{dv} / \mathrm{dt}$ on the output of converter, common in hard switching schemes, generates EMI due to capacitive coupling, and inherently large di/dt can often display interference, leading to concern about the compliance of the respective conducted and radiated interference to EMC regulations. Further, large dv/dt and di/dt result in high switching loss, forming another source of interference. Many methods have been proposed to solve this problem, such as snubbing systems. Soft switching combined with PWM control has been widely applied in many fields and is suggested to be applied to the systems of aircraft power conversion.

\subsection{Electromagnetic Shielding}

Electromagnetic shielding is the process of limiting the penetration of electromagnetic fields via radiation, by blocking them with a barrier made of conductive material. Typically, it is applied to enclosures to separate electrical devices from the "outside world", and to cables to separate wires from the environment through which the cables run. Electromagnetic shielding used to block radio frequency electromagnetic radiation is also known as $\mathrm{RF}$ (Radio Frequency, about $3 \mathrm{kHz}$ to $300 \mathrm{GHz}$ ) shielding.

\subsection{Frequency Modulation}

Random modulation is a new method to reduce EMI proposed in the recent years. Random modulation means that the switch frequency is changed according to a given random signal. Hence the total energy is spread over a wider frequency band .In other words, the harmonic peaks appearing in the frequency band, when the converters operate in periodic mode, can be smoothed over the whole frequency band. The reduced peaks imply that EMI is thus suppressed. There are two main limitations to random modulation: one is that real random signals are difficult to generate in practice, and the other one is that random frequency makes the parameter design of converters difficult, since the parameter design is based on the frequency. For example, when a converter operates with frequency $\mathrm{f}$, the equivalent inductance is $2 \mathrm{pi} * \mathrm{f} * \mathrm{~L}$. Due to the difficulty of obtaining real random signals, pseudo-random signals can be used, leading to the term pseudo random modulation for the method.

\section{SIMULATION RESULTS}

The simulations are done using Advanced Design System(ADS) and the waveforms are plotted. Here we have done two topologies. One is $28 \mathrm{~V}$ to $5 \mathrm{~V}$ topology and the other is $28 \mathrm{~V}$ to combined +15 and $-15 \mathrm{~V}$ topology. Coupled inductors are used to reverse the polarity. The waveforms are given below.

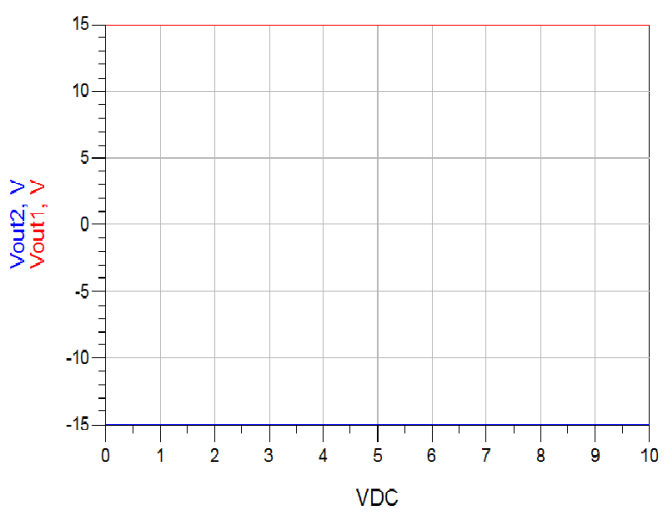

Fig-3: $28 \mathrm{~V}$ to $+15 \mathrm{~V}$ and $-15 \mathrm{~V}$ in a single topology

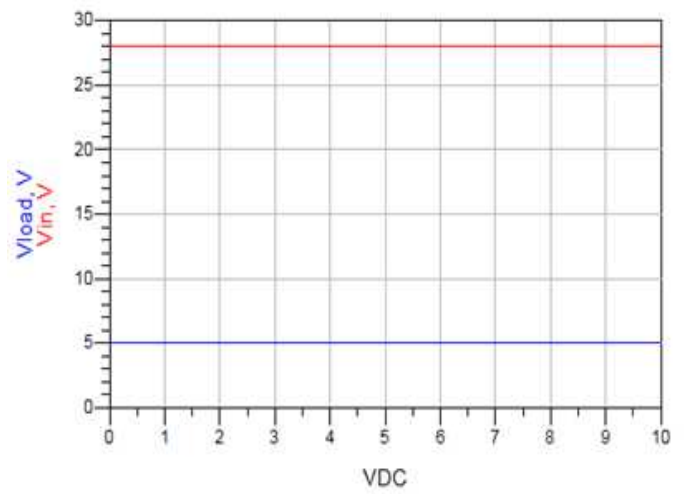

Fig-4: $28 \mathrm{~V}$ to $+5 \mathrm{~V}$ topology

\section{CONCLUSIONS}

By this project, an EMI-free DC-DC Forward converter is designed with a required specification which is compatible for aerospace applications. Different topologies are implemented in the power converter to make the converter free of interferenceand in compliance with MIL-461. A theoretical simulation of the converter design is done using Advanced System Design(ADS). The hardware design should be matching with the simulations that has done earlier. The Radiated Emission(RE) measurement tests are carried out in the microwave anechoic chamber. Even though the EMI is minimized, we cannot remove it completely and what we can do is to remove the EMI as possible as we can. 


\section{REFERENCES}

[1]. A. A. Pereira, E. A. A Coelho, V. J. Farias, L. C. DeFreitas, " A New ZC-ZVS Forward converter ", IEEE,APEC, 1996,pp:482-486

[2]. E.S.Kim, K Y. Joe, M H. Kye,YH. Kim, B. D Yoon, " An Improved ZVZCS PWM FB DC/DCConverter Using Energy Recovery Snubber", IEEE, APEC, 1997, pp:1014-1019

[3]. Eun-Soo Kim, Kee-Yeon Joe, Young-Bok Byun, "An Improved Soft Switching Forward DC/DC Converter UsingEnergy Recovery Snubber",IEEE 1997, pp:46-49

[4]. Jonghoon Kim and Joungho Kim Piljung Jun, "Dithered Timing Spread Spectrum Clock Generationfor Reduction of Electromagnetic Radiated Emissionfrom High-speed Digital System”,2002 IEEE ,pp:413-418.

[5]. J. A. C Pinto, A. A. Pereira, V. J. Farias, L. C. De Freitas, " A New Boost Converter Using A Non- Dissipative Snubber", IEEE, APEC, 1996.

[6]. Keith B. Hardin John T. Fessler Donald R. Bush,"Spread Spectrum Clock Generation for theReduction of Radiated Emissions“",1994 IEEE, pp:227-231

[7]. OlivierTrescases, Guowen Wei, AleksandarProdic', Wai Tung Ng, "An EMI Reduction Technique For Digitally Controlled Smps", Ieee Transactions On Power Electronics, Vol. 22, No. 4, July 2007, pp:1560-1565

[8]. T.Ninomiya, T. Tanaka, K. Harada, "Analysis andOptimization of a Non-dissipative LC Tum-OffSnubber", ieee transactions on power electronics, vol. 3, no. 2, april 1988, pp:147-156

[9]. Yoonjae Lee and Raj Mittra, "Electromagnetic Interference Mitigation by Using a Spread-Spectrum Approach", IEEE Transactions On Electromagnetic Compatibility, Vol. 44, No. 2, May 2002, pp:380-385

\section{BIOGRAPHIES}

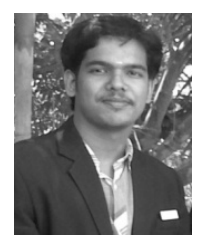

Jithu Unnikrishnan received his B.E degree in Electronics and Communication Engineering from Dhanalakshmi Srinivasan college of engineering and technology, Tamilnadu in 2012.Currentlyhe is pursuing his M.Tech degree in Wireless Technology at Toc $\mathrm{H}$ institute of Science and technology. His area of interest includes Electromagnetic interference and compatibility and wireless communication.

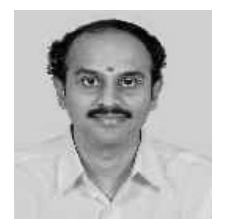

OM Saji Chandrachood achieved his B.E degree in Electronics and Communication from National Engineering college, in 2005. He has done his M.E in Applied Electronics from Noorul Islam college of Engineering, Anna University in 2007 and joined Space Applications Centre (SAC), ISRO in the year 2008. He was working with Indian Regional Navigation Satellite System (IRNSS) project and got transferred to Vikram Sarabhai Space Centre (VSSC) in the year 2010. Currently he is working as Deputy Manager, EMI test facility, RF Systems Group, VSSC responsible for EMI testing of electronic packages of Launch vehicles, satellites and ground based systems.

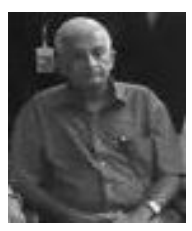

Prof.A.K Prakash received his B.Tech. in Electronics and from IIT Madras and M.Tech in Communication engineering from IIT Mumbai. He is currently associated with Toc $\mathrm{H}$ Institute of Science and Technology, Kerala as a Professor in the department of Electronics and Communication. His areas of interests include Radar systems, Sonar systems, Communication systems.

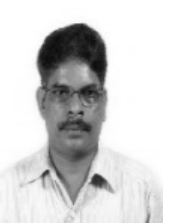

K K Mukundan obtained B.Tech in Electronics Engineering from Regional Engineering College, Calicut, Kerala in August 1985. He joined Space Physics Laboratory, Vikram Sarabhai Space Centre, Trivandrum in September 1985. He was working on phased array antennas. He has designed and developed antenna systems for VHF radar working at 54.95 $\mathrm{MHz}, \mathrm{HF}$ radar working at $18 / 9 \mathrm{MHz}$ and Partial reflection Radar working at $2.5 \mathrm{MHz}$ of Space Physics Laboratory. He is a member of the team who deviced a novel concept of feeding phased array antennas called Interlaced Phased Array (IPA) concept which reduces the number of feed points and phase shifters drastically. He was transferred to RF systems group of avionics entity, VSSC in 2001 and presently he is working as Head Antenna Design and RF facilities division(ADRFD)and responsible for the realization of antenna systems for launch vehicles, spacecrafts and ground based systems. 